\section{An omnibus for the immunologist}

A.J. Munro

Fundamental Immunology.

Edited by William E. Paul.

Raven: 1984. Pp.809. $\$ 80$.

OVER the past few years there has been an explosion of information pertinent to immunologists. The advent of recombinant DNA technology, monoclonal antibodies, and the cloning of functional subsets of lymphocytes has greatly accelerated the rate at which new facts are discovered and has made possible experiments that were beer talk a few years ago.

This presents acute problems for authors or editors of textbooks, who may tackle the difficulties in one of two ways. The first is to produce a relatively short, inexpensive volume attempting a synthesis of the subject and aimed at the undergraduate. The alternative is to compete with the growing number of review journals and book series, such as Immunology Today and Annual Reviews in Immunology, and produce a more detailed, advanced textbook suitable for postgraduates. This second approach requires a firm editor to ensure uniformity of style and a balanced coverage of the subject, but the risk is that the end result will be a cumbersome book that prices itself out of markets.

Fundamental Immunology is a textbook of this latter sort. It is a big book in every sense of the word; in thirty chapters, the fifty two contributors have produced more than half a million words and over three thousand references and there are still areas of the subject that they have not discussed. Overall, the declared emphasis is on basic immunology: the molecules and cells that make up the immune system and how they interact with each other. As to be expected, there is very little discussion of any of the clinical aspects of immunology. Most of the chapters are very well written in an attractive discursive style and make sensible use of original data to illustrate key points.

The best section of the book covers the major histocompatibility complex and its role in the immune response: one of the most complicated parts of immunology. The clarity and coherence of this section may stem from the fact that all four chapters were written by colleagues working in the same institute. Some of the chapters include a few 1983 references but the bulk of the text seems to have been written in 1982 or early 1983; with such a rapidly moving subject, it is unwise for the publisher to promote the volume as "the definitive textbook" as certain chapters already look dated.

Nonetheless, there is no doubt that this book is a success. By providing in one volume a well balanced set of articles cover- ing most aspects of basic immunology, it contributes much more to the teaching of the subject than review journals. Further, the editor has taken great pains to ensure that the book can be approached by students taking advanced courses. The only weak aspect is the index which is inadequate for a volume of this size.

My main complaint, however, is that the book costs so much and I suspect that the publisher is aiming primarily at the library market. Surely the costs could have been kept down; the book would have benefited from being $15 \%$ to $20 \%$ shorter, and is it really sensible to use such high quality paper for a volume that will be superseded in five or six years? However, the high price should not be allowed to deter libraries from buying one or several copies of this useful book.

A.J. Munro is Reader in Immunology in the Department of Pathology, University of Cambridge.

\section{Source of anomalies}

Peter D. Marshall

Earthquakes, Tides, Unidentified Sounds and Related Phenomena: A Catalog of Geophysical Anomalies.

Compiled by William R. Corliss.

The Sourcebook Project, PO Box 107, Glen Arm, MD 21057: 1983. Pp.214. $\$ 12.95$.

HOW EASY it is to pick up a book, scan the title and arrive at a judgement of its contents without reading further. This could easily happen with Earthquakes, Tides, Unidentified Sounds and Related Phenomena. For can such a vast subject be comprehensively covered in a volume of just over 200 pages? The answer is, of course, no. But this in fact is only one of four volumes by Corliss devoted to geophysical phenomena. Eventually there will be at least 25 similar volumes cataloguing numerous "anomalies" observed in nature, all published under the auspices of the Sourcebook Project.

What Corliss is attempting to do as part of the Sourcebook Project is to scour the English-language scientific and semiscientific literature - mainly material published in the United States and Britain over the past 150 years - to extract, assess and categorize all observed and reported anomalous phenomena with particular emphasis on those not readily explained by prevailing scientific theories. Corliss's aims are to collect and consolidate much of the unknown and poorly explained to facilitate future research and explanation. Such a task demands not only a wide scientific knowledge but considerable foresight and planning as to how the phenomena should be categorized and presented in an easily accessible form. If all succeeding volumes of the catalogue are as well organized as this one on geophysics, then the work as a whole will satisfy these requirements.

The plan for the series is to classify each anomaly initially into subject matter (e.g. geophysical, chemical, astronomical etc.). Subsequent classifications are determined by properties directly attributable to the anomaly, e.g. sound, magnetic and so forth. A simple mnemonic lettering system (e.g. $\mathrm{G}=$ Geophysical) followed by a numeral is used to uniquely identify and catalogue the anomaly; this makes indexing and cross-referencing a relatively simple procedure. Once classified, the anomaly is further rated on a scale of 1 to 4 on the basis of the quality of the observations, to indicate whether, in the author's opinion, there is a high probability that the anomaly was a true phenomenon or not and whether the data are unacceptably poor. A similar scale is used to rate the quality of the explanation of the anomaly.

The phenomena catalogued in this latest volume are collected under three principal headings: the hydrosphere, earthquakes and unusual sounds in nature. All observations of water-related phenomena such as typhoon waves, geysers, tidal bores, ocean turbulence and freak wave activity are included in the hydrosphere section. The abundance of strange phenomena before, during and after earthquakes are adequately covered, though many scientists would argue with some of the conclusions presented here. Finally, the section on acoustic phenomena lists reports of strange acoustic emissions including those associated with auroral activity and winds in the desert.

For a catalogue, the book makes surprisingly interesting reading. Each phenomenon is fully described, occasionally with diagrams and figures. Additional background information, assessments and possible explanations are given as well as specific details of similar or related phenomena. Numerous observations are reported in detail with an extensive list of references dating back to the mid-nineteenth century. Such aged documentation regrettably makes verification of some observations difficult.

My main criticism of the project is one of semantics. It would have been better if, throughout the catalogue, each event was described as a phenomenon rather than an anomaly; what the author has described as an anomaly may, in reality, be a normal and predictable occurrence albeit an extremely rare one. There are inevitably other shortcomings, which may be due to a lack of consultation with appropriate experts. But on the whole Corliss has been successful in producing a book which may be of interest to both the casual browser and the specialist.

Peter D. Marshall is a Principal Scientific Officer in the Seismology Division of the Ministry of Defence, Aldermaston. 